\title{
ASPECTS OF ELECTRODYNAMICS OF ELECTROCHEMICAL SYSTEMS
}

\author{
R. R. DOGONADZE, A. A. KORNYSHEV, A. M. KUZNETSOV and T. A. MARSAGISHVILI \\ Institute of Electrochemistry of the Academy of Sciences of the USSR \\ Leninskij Prospect 31 Moscow V-71, USSR
}

\begin{abstract}
Résumé. - On discute les bases du formalisme de la réponse électromagnétique, et le rôle đes modes collectifs en électrochimie interfaciale. Ces concepts sont utilisés pour développer un point de vue sur les problèmes optico-électrochimiques des modes collectifs ou mettant en jeu une particule individuelle, en volume et à l'interface, ainsi que pour réunir quelques idées sur les effets physiques possibles.

Abstract. - Fundamentals of electromagnetic response formalism and the role of collective modes in interfacial electrochemistry are discussed. These concepts are used to derive a point of view on the optico-electrochemical problems of bulk and interfacial collective and single-particle modes and to collect some ideas on the possible physical effects.
\end{abstract}

1. Introduction. - Interfacial electrochemistry at present draws considerable attention of physists and chemists working in different fields. In fact, what is usually called the electrochemical system (ES) is composed of electrodes, made of metallic or semiconductor materials or of metals covered by oxide films, and of electrolyte. So, the electrochemistry is directly coupled with practically all the main branches of physics of condensed matter and is strongly affected by their development.

The recent progress in experimental and theoretical methods of surface science (e.g. electromagnetic wave, electron and neutron diffraction spectroscopy, and the dynamic theory of surfaces $[1,2])$ has given a new impuls to interfacial electrochemistry. However, the ES contains components much more complicated than met in surface physics experiments $[3,4]$ (except for electromodulation spectroscopy [5], traditionally connected with interfacial electrochemistry, but having many unsolved interpretational questions). Thus we may speak only about the first steps in applications of the methods to ES.

Dealing with ES we encounter typical many-body problems. In this paper we shall discuss different collective motions in ES.

External perturbation probes may provide information on real processes in the system, if they are coupled with the same type of fluctuation. Thus, the Rayleigh scattering of light in a solution [6], coupled with the excitation of translational (or rotational) motions give a valuable information on the diffusion in ES. Other types of probes are required for information on the elementary act of charge transfer, and the radiationless transition involving the collective excitations interacting with charged particles (pola- rization waves). Appropriate probes here are the electromagnetic wave and electron diffraction experiments, and this type of collective motions will be the main subject of this paper.

2. Electromagnetic Green-function formalism. - The most general way to describe electromagnetic modes in the medium is provided by the Green-function formalism [7]. In classical mechanics the motion of a particle in an external field can be described in terms of the trajectory of the particle. In quantum mechanics the evolution of a single-particle wave-packet interacting with an external field can be formulated in terms of trajectory intergrals [8], or propagators, relating the wave-functions in the space-time point $\mathbf{r}$, $t$ with the point $\mathbf{r}^{\prime}, t^{\prime}$. The behaviour of a particle interacting with the many-body assembly is also described by a propagator, the single-particle Greenfunction.

However, the description of a many-body system in terms of single-particle Green-functions is not complete. The latter describes possible excitation of the system due to adding one particle into the point $r$ at the moment $t$ with a subsequent removal of a particle in $\mathbf{r}^{\prime}, t^{\prime}$ space-time point. More complete information is contained in the two-particle Green-function, which incorporates elementary excitations in the system due to an addition of two particles in the points $\mathbf{r}_{1}, t_{1}$ and $\mathbf{r}_{2}, t_{2}$ with the following removal in the points $\mathbf{r}_{1}^{\prime}, t_{1}^{\prime}$ and $\mathbf{r}_{2}^{\prime}, t_{2}^{\prime}$. Analogously, higher many-particle Green-functions are introduced. The total information is given, strictly speaking, only by the whole set of many-particle Green-functions. In many problems, however, it is sufficient to know only single-particle (or two-particle) Green-functions. In fact, let a weak 
electromagnetic field, produced by a test current $\mathbf{j}^{\mathrm{ex}}(\mathbf{r}, t)$ be superposed on the system. The quantumstatistical average of the electromagnetic field calculated in first-order perturbation theory is then linear in the current

$$
\begin{aligned}
& <A_{\alpha}(\mathbf{r}, t)>= \\
& \quad-\frac{1}{c} \int \mathrm{d} \mathbf{r}^{\prime} \times \int_{-\infty}^{\infty} \mathrm{d} t^{\prime} D_{\alpha \beta}^{\mathrm{R}}\left(\mathbf{r}, \mathbf{r}^{\prime}, t-t^{\prime}\right) j_{\beta}^{\operatorname{ex}}\left(\mathbf{r}^{\prime}, t^{\prime}\right)
\end{aligned}
$$

where $D^{\mathrm{R}}$ is the single particle retarded electromagnetic Green-function [7], i. e.

$$
\begin{aligned}
D_{\alpha \beta}^{\mathrm{R}}\left(\mathbf{r}, \mathbf{r}^{\prime}, t-t^{\prime}\right)= & -\frac{i}{\hbar} \theta\left(t-t^{\prime}\right) \\
& \times<\left[A_{\alpha}(\mathbf{r}, t), A_{\beta}\left(\mathbf{r}^{\prime}, t^{\prime}\right)\right]>
\end{aligned}
$$

$\mathbf{A}(\mathbf{r}, t)$, the vector-potential, is associated with the electro-magnetic field by the relation

$$
\mathbf{E}(\mathbf{r}, t)=-\frac{1}{c} \frac{\partial \mathbf{A}(\mathbf{r}, t)}{\partial t} ; \mathbf{H}(\mathbf{r}, t)=\operatorname{rot} \mathbf{A}(\mathbf{r}, t) \text {. }
$$

Only the single-particle Green-function figures in equation (2.1), since within first order in the (small) $j^{\text {ex }}$ only the processes of emission or absorption of one photon are taken into account. The next correction to equation (2.1) contains the two-particle Greenfunction as the coefficient in the term quadratic in $\mathbf{j}^{\mathrm{ex}}$, corresponding to processes with participation of two photons. By analogy one may consider higherorder nonlinear corrections.

In the linear approximation, any electromagnetic characteristic of the system can be expressed through the Green-function $D^{R}$. For instance, the problem of electromagnetic wave diffraction in an arbitrary system is reduced to the calculation of $D^{\mathrm{R}}$. In fact, in the transparent uniform medium the average vector potential $\left\langle\mathbf{A}^{0}\right\rangle$ is associated with the same testcharge current $\mathbf{j}^{\mathrm{ex}}$ by the relation (2.1), but with the kernel

$$
D_{\alpha \beta}^{\mathrm{R}^{0}}\left(\mathbf{r}-\mathbf{r}^{\prime}, t-t^{\prime}\right) \text {. }
$$

If the source, imbedded in a uniform transparent medium, produces an electromagnetic wave $\mathbf{A}^{0}$ which is then scattered by nonuniform loss regions, or impurity objects, the vector potential of the scattered wave $\mathbf{A}$ may be expressed through $\mathbf{A}^{0}$ of the incident wave.

$$
\begin{array}{r}
A_{\alpha}(\mathbf{r}, t)=\int \mathrm{d} \mathbf{r}^{\prime} \mathrm{d} t^{\prime} \int \mathrm{d} \mathbf{r}^{\prime \prime} \mathrm{d} t^{\prime \prime} D_{\alpha \gamma}^{\mathrm{R}}\left(\mathbf{r}, \mathbf{r}^{\prime \prime}, t-t^{\prime \prime}\right) \times \\
\times D_{\gamma \beta}^{\mathrm{R} 0-1}\left(\mathbf{r}^{\prime \prime}, \mathbf{r}^{\prime}, t^{\prime \prime}-t^{\prime}\right) A_{\beta}^{0}\left(\mathbf{r}^{\prime}, t^{\prime}\right)
\end{array}
$$

or in Fourier components :

$$
A_{\alpha}(\mathbf{k}, \omega)=\sum_{\beta, \mathbf{k}_{0}} D_{\alpha \gamma}^{\mathbf{R}}\left(\mathbf{k}, \mathbf{k}_{0}, \omega\right) D_{\gamma \beta}^{\mathrm{R} 0-1}\left(\mathbf{k}_{0}, \omega\right) A_{\beta}^{0}\left(\mathbf{k}_{0}, \omega\right)
$$

where $D^{\mathrm{R}^{0}} \cdot D^{\mathrm{R}^{0-1}}=1$. The knowledge of $\mathbf{A}(\mathbf{k}, \omega)$ provides any electrodynamic characteristics of the scattered radiation. In our condition of gauge invariance

$$
D_{\alpha \beta}^{\mathrm{R}^{0-1}}(\mathbf{k}, \omega)=\delta_{\alpha \beta} \frac{1}{4 \pi}\left(\varepsilon_{\perp}^{0}(\omega, k) \frac{\omega^{2}}{c^{2}}-k^{2}\right)
$$

and it is only left to determine $D_{\alpha \beta}^{R}\left(\mathbf{k}, \mathbf{k}_{0}, \omega\right)$.

It should be emphasized that no assumptions on the macroscopic character has been involved in equations (2.4) or (2.5). They describe any system at any scale. But the determination of the Green-function $D^{\mathrm{R}}$ is, in general, a very complicated problem. However, in the limit, when, the radiation wavelength is much greater than the interparticle separation in the medium $D^{R}$ is shown to be the Green-function of Maxwell's equations (subject to appropriate boundary condition) [7]. It can thus be calculated on the basis of macroscopic theory and expressed through the dielectric function $\varepsilon_{\alpha \beta}\left(\omega, \mathbf{r}, \mathbf{r}^{\prime}\right)$ [7]. Examples of simple expressions for $D^{\mathrm{R}}$ through the permittivities of two contacting media (with no spatial dispersion) or three layer systems can be found in $[7,9,10]$.

In the short-wave limit, $D^{\mathrm{R}}$ does not obey Maxwell's equations and should be found from a micro-theory. It might be noted that the dielectric function $\varepsilon_{\alpha \beta}\left(\mathbf{r}, \mathbf{r}^{\prime}, \omega\right)$ is not necessarily a macroscopic characteristic : it can be defined through the correlator of the polarizationfluctuation in any quantum system. However, the relation between $\widehat{D}^{\mathrm{R}}$ and $\widehat{\varepsilon}$ at the microscopic scale is not defined by Maxwell's equation. Thus, in microsystems, it may be convenient not to introduce dielectric-functions at all, but start directly with the calculation of the Green-function, which itself gives the electrodynamic (optical), as well as thermodynamic [7] characteristics.

A convenient formalism is available for the calculation of the Green-functions. The retarded temporal Green-functions are related with the so-called temperature Green-functions [7]

$$
D_{\alpha \beta}\left(\mathbf{r}, \mathbf{r}^{\prime}, \omega_{n}\right)=D_{\alpha \beta}^{\mathrm{R}}\left(\mathbf{r}, \mathbf{r}^{\prime}, i\left|\omega_{n}\right|\right)
$$

defined for a discrete variety of frequencies $\omega_{n}$ (e. g., for Bose-systems $\hbar \omega_{n}=2 n \pi k T, n$ is an integer). For $D$ a diagram technique is developed [7]. $D$ obeys the Dyson equation [7]

$$
\begin{aligned}
D_{\alpha \beta}\left(\mathbf{r}, \mathbf{r}^{\prime}, \omega_{n}\right)= & D_{\alpha \beta}^{0}\left(\mathbf{r}-\mathbf{r}^{\prime}, \omega_{n}\right)+ \\
& +\int \mathrm{d} \mathbf{r}^{\prime \prime} \mathrm{d} \mathbf{r}^{\prime \prime \prime} D_{\alpha \gamma}^{0}\left(\mathbf{r}-\mathbf{r}^{\prime \prime}, \omega_{n}\right) \\
& \times \pi_{\gamma \delta}\left(\mathbf{r}^{\prime \prime}, \mathbf{r}^{\prime \prime \prime}, \omega_{n}\right) D_{\delta \beta}\left(\mathbf{r}^{\prime \prime \prime}, \mathbf{r}^{\prime}, \omega_{n}\right) .
\end{aligned}
$$

In the long-wave limit the polarization operator $\pi$ contains the information on the medium only. (In the long-wavelength limit $\pi$ is expressed through the permittivity as a function of $\omega=i\left|\omega_{n}\right|$ [7].) There are also other spectral approximations which make $\pi$ independent of $D$ such as the random phase approximation, and the self-consistent field, for which $\pi$ does not contain photon lines. 
Inserting in equation (2.5) an expression for $D$ through the frequency-dependent permittivities of the two media in contact (via a plane interface) one obtains the usual Fresnel formulae. Furthermore, an additional signal associated with the presence of an impurity, adsorbed at the interface, is described by equation (2.5) with $D$ replaced by $\Delta D=D^{1}-D$, where $D$ and $D^{1}$ are the Green-functions of the pure twocomponent system and the two-component system with impurity, respectively. This approach provides the results obtained in reference [11] for s-polarized waves, together with more complicated results subject to p-polarization. However, it is a tedious task to obtain the Fresnel-like equations [12] from (2.5), i. e. to express diffraction characteristics through permittivities. It is more convenient to start directly with Maxwell's equations. This is the reason why the dielectric description of media was so profitable. The value of equation (2.5) is not only its generality (it covers the cases when $D$ is simply expressed through permittivities and when $D$ should be calculated from micro-models) ; but also the fact that it is the most convenient formula provided that $D$ has been calculated directly.

3. Dielectric response of uniform systems. - We consider in this section uniform isotropic dielectrics and discuss some aspects od dielectric formalism, which, as indicated above, in some cases is more convenient than the direct electromagnetic Greenfunction approach.

We may regard the dielectric as a fluctuating polarization field $\mathbf{P}$. The linear response of the dielectric to an external electric field $\mathbf{E}^{0}$ (the vacuum value) is defined by the relation

$$
\begin{aligned}
& <P_{\alpha}(\mathbf{r}, t)>= \\
& \sum_{\beta} \int \mathrm{d} \mathbf{r}^{\prime} \times \int_{-\infty}^{\infty} \mathrm{d} t^{\prime} \chi_{\alpha \beta}\left(t-t^{\prime}, \mathbf{r}-\mathbf{r}^{\prime}\right) E_{\beta}^{0}\left(\mathbf{r}^{\prime}, t^{\prime}\right)
\end{aligned}
$$

or in Fourier transforms in space $(\mathbf{k})$ and time $(\omega)$

$$
\left\langle P_{\alpha}(\mathbf{k}, \omega)\right\rangle=\sum_{\beta} \chi_{\alpha \beta}(\mathbf{k}, \omega) E_{\beta}^{0}(\mathbf{k}, \omega) .
$$

The intergral operator $\chi$ is called the linear response function (permeability).

The nonlocal nature of the response with respect to time $\left(E^{0}\right.$ in the previous moments $t^{\prime}$ define the value of polarization at a time $t$ ) is caused by the inertia of the movements of the medium particles and referred to as the time dispersion [13]. The nonlocal nature in space (the value of the field in different points $\mathbf{r}^{\prime}$ determine the polarization at a point $\mathbf{r}$ ) is due to the structure of the dielectric and referred to as spatial dispersion [13]. It is important that we cannot speak about spatial structure independently of the time structure. In fact, different degrees of freedom of the system are correlated in a different manner. For example, the polarization fluctuation in a dipolar liquid is composed of different contributions, in particular hindered rotation of dipoles, much faster movements due to infrared intramolecular vibration of dipoles, and high-frequency electronic transitions changing the dipole moment. Each of these modes has a separate correlation range. Thus, rotations are correlated by a hydrogen-bonding chain (if it is present in the system) or dipole forces; vibration by short-range repulsion forces, and electronic polarization by exchange interaction.

If we apply the factoriation anzatz, according to which all high-order correlation functions are expressed through the pair correlation, all the properties of the dielectric can be expressed through $\chi(\omega, \mathbf{k})$, or through the dielectric function

$$
\varepsilon_{\alpha \beta}(\omega, \mathbf{k}) \equiv \delta_{\alpha \beta}+4 \pi \chi_{\alpha \beta}(\omega, \mathbf{k}) .
$$

However, application of the factorization procedure is equivalent to the self-consistent field approach [16] and may be justified only for very simple one-component systems (e. g. a free electron gas [17).

The aim of the response formalism is to express the features of the phenomena proceeding in the medium through a universal response function. This function could next be obtained from independent experiments or from model calculation. Within this formalism, and for a definite Hamiltonian of the system, a response function, $\varepsilon(\omega, k)$, is calculated, and relationships (sum rules) between the Hamiltonian parameters and the quantities coupling the bound charge fluctuations and the normal coordinates, etablished. Afterwards, in the calculation of different thermodynamic properties and charge-transfer kinetic characteristics these sum rules are applied to express the quantities of interest through $\varepsilon(\omega, k)$. This approach has been applied to the theory of fast electron spectroscopy of solids [24], small polaron theory [25], polar solvents [21], the theory of Van-der-Waals forces [26], etc.

Let us now consider some expressions for the dielectric function $\varepsilon_{\alpha \beta}(\omega, \mathbf{k})$ of a uniform medium. The Fourier transform of the polarization field.

$$
\mathbf{P}_{\mathbf{k}}=\int \mathrm{d} \mathbf{r} \mathrm{e}^{-i \mathbf{k r}} \hat{\mathbf{P}}(\mathbf{r}) \equiv \frac{\mathbf{k}}{|k|} \hat{P}_{\mathbf{k}}^{\|}+\widehat{\tau}_{\mathbf{k}} \hat{P}_{\mathbf{k}}^{\perp}
$$

in the quasiparticle representation is composed of collective excitations of Bose-type of different $(v)$ branches $\left({ }^{1}\right)$

$$
\widehat{P}_{\mathbf{b}}^{\|, \perp}=2^{-1 / 2} \sum_{v} B_{v}^{\|, \perp}(k)\left(\omega_{v k}^{\|, \perp}\right)^{-1 / 2}\left(\hat{a}_{v k}^{\|, \perp}+a_{v-k}^{+\|, \perp}\right)
$$

while the Hamiltonian of the system is written as

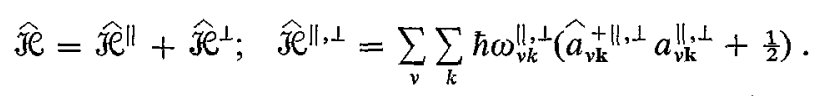

(1) Analogical many-branches representation for nonpolar disordered system see, e. g., in refs. [22, 23]. 
Here $\hat{a}_{v k}$ and $\hat{a}_{v k}^{+}$are the creation and annihilation operator for a boson with wave vector $k$ of the $v$-th type, $\hbar \omega_{v k}$ the energy of the quasiparticle, $B_{v}(k)-a$ quantity analogous to the oscillator strength; the superscripts $\|, \perp$ correspond to longitudinal and transversal modes. By a standard procedure [14] one may obtain the response function relating $\mathbf{P}$ and $\mathbf{E}_{0}$, i. e.

$$
\chi^{\|, \perp}(\omega, k)=\sum_{v} \frac{\left[B_{v}^{\|, \perp}(k)\right]^{2}}{\omega_{v k}^{2}-\omega^{2}-i \delta \omega /|\omega|} .
$$

Since in a uniform medium, with no external charges, the external field $\mathbf{E}_{0}$ is identical to induction vector D [27]

$$
\chi(\omega, k)=\frac{\left\langle\hat{\mathbf{P}}(\mathbf{r}, t)>_{\mathbf{k}, \omega}\right.}{D(\mathbf{k}, \omega)}=\frac{1}{4 \pi}\left[1-\varepsilon^{-1}(\omega, k)\right] .
$$

Equation (3.6) gives the relation between the response function $\chi(\omega, k)$ and $\varepsilon(\omega, k)$.

In the case $B_{v}(k)=\delta_{v 0} B_{0}+\delta_{v \infty} B_{\infty}$, where $B_{0}=$ const., $B_{\infty}=$ const. and $\omega_{0 k}=\omega_{0}=$ const., $\omega_{\infty k}=\omega_{\infty}=$ const., equation (3.5) gives the simple result

$$
\begin{aligned}
& \chi(\omega, k)=\frac{B_{0}^{2}}{\omega_{0}^{2}-\omega^{2}-i \delta \omega /|\omega|}+ \\
& \quad+\frac{B_{\infty}^{2}}{\omega_{\infty}^{2}-\omega^{2}-i \delta \omega /|\omega|}=\chi(\omega)
\end{aligned}
$$

which corresponds to a response function of two harmonic oscillators. This expression is frequently used for the description of infrared resonance due to optical phonons in ionic crystals or exitons in semiconductors and organic solids in the absence of damping and spatial dispersion. If $\omega_{0} \ll \omega_{\infty}$, for the frequencies close to $\omega_{0}$-resonance (but $\omega \neq \omega_{0}$ ), using equalities,

$$
\begin{aligned}
B_{\infty}^{2} / \omega_{\infty}^{2} & =\left[1-\varepsilon_{\infty}^{-1}\right] / 4 \pi ; \\
B_{0}^{2} / \omega_{0}^{2} & =\left[\varepsilon_{\infty}^{-1}-\varepsilon_{0}^{-1}\right] / 4 \pi
\end{aligned}
$$

one may write

$$
\varepsilon(\omega)=\varepsilon_{\infty} \frac{\omega_{0}^{2}-\omega^{2}}{\omega_{t}^{2}-\omega^{2}}
$$

where $\omega_{0}$ and

$$
\omega_{\mathrm{t}}=\left(\frac{\varepsilon_{\infty}}{\varepsilon_{0}}\right)^{1 / 2} \omega_{0} \quad\left(\varepsilon_{0}=\varepsilon(\omega=0)\right)
$$

are the frequencies of longitudinal and transversal optical phonons, respectively [28].

If the spatial dispersion within $\omega_{0}$ resonance is taken into account, then

$$
\begin{aligned}
& {\left[\varepsilon_{\infty}^{-1}-\varepsilon^{-1}(k, \omega)\right] / 4 \pi=} \\
& \quad=B_{0}^{2}(k) /\left[\omega_{0}^{2}(k)-\omega^{2}-i \delta \omega /|\omega|\right] .
\end{aligned}
$$

Models with weak spatial dispersion [29] have fre- quently been analysed. Thus $B_{0}(k)$ and $\omega_{0}^{2}(k)$ are slowly varying functions of $k$, in particular

$$
B_{0}(k)=B_{0}^{2}+A k^{2}, \quad \omega_{0}^{2}(k)=\omega_{0}^{2}+D k^{2} .
$$

Having put

$$
B_{0}^{2}(k) / \omega_{0}^{2}(k)=\left[\varepsilon_{\infty}^{-1}-\varepsilon^{-1}(k)\right] / 4 \pi,
$$

we may write close to resonance $\left(\omega \neq \omega_{0 k}\right)$

$$
\begin{aligned}
\varepsilon_{\|, \perp}(\omega, k) & =\varepsilon_{\infty} \frac{\omega_{0 \|, \perp}^{2}(k)-\omega^{2}}{\omega_{\|, \perp}^{2}(k)-\omega^{2}} \\
\omega_{\|, \perp}^{2}(k) & =\frac{\varepsilon_{\infty}}{\varepsilon_{\|, \perp}(\omega=0, k)} \omega_{0 \|, \perp}^{2}(k) .
\end{aligned}
$$

An appropriate choice of $B_{v}^{2}(k) /\left|\frac{\delta \omega_{v k}}{\partial v}\right|$, provides resonance with damping.

$$
\varepsilon(\omega)=\varepsilon_{0}+\left(\varepsilon_{0}-\varepsilon_{\infty}\right) \frac{\omega_{r}^{2}+\Gamma_{r}^{2}-i \Gamma_{r} \omega}{\omega_{r}^{2}-\left(\omega+i \Gamma_{r}\right)^{2}}
$$

where $\varepsilon_{0}$ and $\varepsilon_{\infty}$ are the values of the dielectric function in the transparency bands confirming the resonance from the low frequency and high frequency sides, respectively. Similarly, the pure Debye dispersion may be obtained

$$
\varepsilon(\omega)=\varepsilon_{\infty}+\frac{\varepsilon_{0}-\varepsilon_{\infty}}{1-2 i \omega \Gamma_{D}} .
$$

For the case of fluctuations of the concentrations of free charge carriers, $B_{v}(k)$ and $\omega_{v k}^{2}$ can be shown to obey the sum rules [30]

$$
4 \pi \sum_{v}\left[B_{v}(k)\right]^{2} / \omega_{v k}^{2}=x^{2} / k^{2} ; 4 \pi \sum_{v}\left[B_{v}(k)\right]^{2}=\omega_{\mathrm{p}}^{2}
$$

where $x^{-1}$ is the Debye or Thomas Fermi screening distance [31] for classical and degenerate plasma, respectively ; the plasma-frequency

$$
\omega_{p}=\left(\frac{4 \pi n e^{2}}{m}\right)^{1 / 2}
$$

where $n$ is the average concentration of charged particles, and $m$, the mass of the particles.

For dielectrics, analysis of $\varepsilon(\omega, k)$ based on band models is found in ref 85 . Simple analytical approximations for $\varepsilon(\omega, k)$ or $\varepsilon(0, k)$ are given in ref. [32, 34$36]$.

4. Problem of nonuniformity. Dielectric formalism for the systems with interfaces. - The dielectric formulation is much less developed - if it exists - for nonuniform systems. Firstly, $\varepsilon\left(\mathbf{r}, \mathbf{r}^{\prime}, \omega\right)$ and $\chi\left(\mathbf{r}, \mathbf{r}^{\prime}, \omega\right)$ are not functions of $\mathbf{r}-\mathbf{r}^{\prime}$ as in the systems possesing total translational invariance. Thus

$$
<\widehat{P}_{\alpha}(\mathbf{k}, \omega)>=\sum_{\mathbf{k}, \beta} \chi_{\alpha \beta}\left(\mathbf{k}, \mathbf{k}^{\prime}, \omega\right) E_{\beta}^{\mathrm{o}}\left(\mathbf{k}^{\prime}, \omega\right)
$$

so that Fourier-components, $\left\langle\mathbf{P}_{\mathbf{k}}\right\rangle$ and $\mathbf{E}_{\mathrm{k}}^{0}$, with different wave-vectors are coupled. Due to the integral character of relations in $\mathbf{k}$-space, the analysis 
becomes very complicated. But the main problem is associated with the fact that the Green's function $\chi\left(\mathbf{k}, \mathbf{k}^{\prime}, \omega\right)$ of the medium, which may be calculated on the basis of effective Hamiltonians and secondary quantization of polarization, is no longer related with $\varepsilon\left(\mathbf{k}, \mathbf{k}^{\prime}, \omega\right)$ by a simple equality of (3.6) type. In fact, the external field $\mathbf{E}^{0}(\mathbf{k}, \omega)$ is generally not equal to the induction in a nonuniform medium. Then, in order to express the Hamiltonian frequencies and oscillator strength through $\varepsilon\left(\mathbf{k}, \mathbf{k}^{\prime}, \omega\right)$ one should find an operator $\widehat{L}$ which transforms $D$ into $E^{0}$ :

$$
E_{\alpha}^{0}(\mathbf{k}, \omega)=\sum_{x, \gamma} L_{\alpha \gamma}(\omega, \mathbf{k}, x) D_{\gamma}(x, \omega) .
$$

The knowledge of $\widehat{L}$ would thus provide the result for $\hat{\varepsilon}$, since

$$
\left[\widehat{I}-\varepsilon^{-1}\right] / 4 \pi=\widehat{L} \chi
$$

and the form of $\hat{L}$ is determined by the character of the nonuniformity of the system.

The nonuniformity of the system may originate from different reasons. It may be produced by discrete translational invariance of perfect crystals (the problem of $\varepsilon_{\alpha \beta}\left(\mathbf{k}, \mathbf{k}^{\prime}, \omega\right)$ due to crystal structure is discussed in reference [18]). It can be associated with impurities [27] (as in the case of charge transfer between a pair of impurity ions [36], or, it may arise from the presence of interfaces between quasi-uniform media. The effects due to the first two reasons in some cases may not be too significant.

Let us discuss the interfacial effect. First of all, at the contacts of different media or near the vacuum boundary foreign structure defect surface layers are usually present [38]. Their width is, typically, of the same order of magnitude, as the characteristic structure distances of the medium in the bulk. Therefore, as far as the spatial dispersion is involved, the defect layer contribution to the $k, k^{\prime}$ dependence should be taken into account (except for the case, when the phenomenon is defined by regions far enough from the surface).

For a moment let us disregard this surface defect structure (when it is well defined, in some cases a defect layer may be considered as a foreign thin film). Even in this case the $k, k^{\prime}$ dependence takes place due to the breakdown of the translational invariance. The models neglecting the defect structure near interfaces are sometimes called sharp-surface models [39] (SSM). In SSM the boundary itself works as a defect, since it may reflect elementary excitations propagating from the bulk. The manner in which this reflection takes place affects the response of the whole system. Finally, the boundary provides the area where excitation of surface modes is possible.

At present there are three main approaches in the study of the SSM. Firstly, the method of additionnal boundary conditions (ABC's). Since it has been found that the account of spatial dispersion in the bulk of dielectric leads to new normal electromagnetic modes, the simplest idea was to match the bulk solutions from both sides of the boundary, introducing ABC. The work on this subject usually concerned with the wave propagation with the frequencies close to excitonic resonance. When account of spatial dispersion is taken up to the correction of the order of $k^{2}$, two branches of normal modes (at certain conditions) may propagate in the system instead of one normal wave in the case of the neglect of spatial dispersion. Then only one $A B C$ is required. Different authors tried to employ different $A B C$ 's. They were usually reduced to requirements on the value of the components of the polarization vector at the boundary. Pekar [40], who was the first to propose the idea of $A B C$, used

$$
\left.P_{x}\right|_{z=0}=0
$$

( $z$ - is the axis normal to the interface). Hopfield and Thomas [41] proposed more generally

$$
\left.\left\{A P_{x}+B \frac{\mathrm{d} P_{x}}{\mathrm{~d} z}\right\}\right|_{z=0}=0
$$

with $A$ and $B$ being the fitting parameters of the theory. Agranovich and Ginzburg [18] intensively analyzed the most general tensor form of $\mathrm{ABC}$, on the basis of which a phenomenological theory of transmission and reflection can be developed (the components of the tensor being a phenomenological quantity of the theory).

On the other hand, the solution of Maxwell's equations subject to the usual boundary conditions and the true constitutive relation

$$
P_{\alpha}(z, \omega)=\sum_{\beta} \int_{0}^{\infty} \mathrm{d} z^{\prime} \chi_{\alpha \beta}\left(z, z^{\prime}, \omega\right) E_{\beta}\left(z^{\prime}, \omega\right)
$$

does not require any $\mathrm{ABC}$ 's. It will per se provide the arrival of all the branches and define the value of the coefficients, which determine the contribution of each branch to the value of the total field $E$.

In the studies of electromagnetic properties of semiinfinite plasma, it was found that when electrons (or plasma ions) are reflected from the boundary in a diffuse or purely specular manner, then in the case of s-polarized field

$$
P_{x}(z)=\int_{0}^{\infty} \mathrm{d} z^{\prime} \chi_{x x}\left(z, z^{\prime}\right) E_{x}\left(z^{\prime}\right)
$$

( $z$ axis is normal to the boundary) with

$$
\chi_{x x}\left(z, z^{\prime}\right)=\chi_{x x}\left(z-z^{\prime}\right)+\rho \chi_{x x}\left(z+z^{\prime}\right)
$$

where $\rho$ is the portion of particles which are scattered specularly from the boundary, among the total number of particles being reflected. This was the first microscopically justified model, which expressed the very complicated $\left(z, z^{\prime}\right)$ response of a semi-infinite system through the response functions $\left(z-z^{\prime}\right)$ of the bulk. It was applied for the calculation of the anomalous skin-effect, and for purely specular reflection $(\rho=1)$ the famous expression for the depth of penetration of the field into plasma was obtained [20,42]. 


$$
\lambda_{s}=-\frac{1}{\pi} \int_{-\infty}^{+\infty} \mathrm{d} k \frac{1}{\frac{\omega^{2}}{c^{2}} \varepsilon_{\perp}(\omega, k)-k^{2}}
$$

where $\varepsilon_{\perp}(\omega, k)$ is the bulk transversal dielectric function. This result encouraged many authors to study the semi-infinite plasma response. Indeed, for $\rho=1$, having put $E_{x}(-z)=E_{x}(z)$, the equation for $P_{x}$ is transformed to

$$
P_{x}(z)=\int_{-\infty}^{+\infty} \mathrm{d} z \chi_{x x}\left(\left|z-z^{\prime}\right|\right) E_{x}(z),
$$

so that the constitutive relation becomes identical to the case of uniform media, and normal mode Fourier solutions are available. For $\rho=0$ (purely diffuse scattering) the procedure of the solution of the field equations is more complicated since they are reduced to integro-differential equations of the Wiener-Hopf type. The use of Wiener-Hopf techniques makes it possible to obtain the results for a general form of the bulk response. Thereby, an expression for the penetration depth of electromagnetic s-polarized field into semi-infinite plasma has been obtained : $[20,42,43]$

$$
\lambda_{\mathrm{s}}=\left\{\frac{1}{\pi} \int_{0}^{\infty} \mathrm{d} k \ln \left[1-\frac{\omega^{2}}{c^{2} k^{2}} \varepsilon^{\mathrm{tr}}(\omega, k)\right]\right\}^{-1} .
$$

However, for some special cases of $\varepsilon(\omega, k)$ the WienerHopf equation may be reduced to higher order usual differential equations. A method of solution of the case with, $0<\rho<1$ was also developed [44].

The idea of replacing the complicated response of a semi-infinite medium by some combination of functions, describing the bulk response, opened the door of an easy way of analytical treatment of the problem. Soon it became popular in the description of systems other than plasma, such as, e. g. bounded ionic crystals. For instance, Fuchs and Kliever, having firstly analyzed surface impedance of a semi-infinite electron gas in the specular reflection model [45], came to the application of this model to the study of surface optical phonons [46]. The specular reflection model was especially popular, since it provided the most simple analytical procedure [47].

Other authors proposed an alternative treatment, i. e. a simple replacement of $\left(z, z^{\prime}\right)$ response by its bulk analogue $\chi_{\alpha \beta}\left(z-z^{\prime}\right)[19,48]$. For s-polarized field $\mathbf{E}=E_{x} \mathbf{n}_{x}$ it coincides with the approximation $\rho=0$, i. e. the diffuse scattering of plasma particles from the boundary. But this model was applied to dielectrics with no plasma and no particles, being reflected from the boundary. The model was considered as purely phenomenological, and was called the dielectric approximation [48] (DA).

It was clear that none of the models (specular or DA) has any foundation when they are applied to systems other than plasma. At the same time, the analogy between the ABC's assumptions and these models has been established [49].
An attempt to reveal the type of constitutive relation or $\mathrm{ABC}$ on the basis of a specific micro-model of a semi-infinite crystal was made by Agranovich and Judson [50]. For a simple model, they have shown that the constitutive relation of the DA type arises only at a single occasional frequency. For all other frequencies the kernel turned out to be a function of $z, z^{\prime}$. This paper induced a storm of criticisms against the bulk dielectric function approaches. Since then other models also supported its arguments [51]. Moreover, on the basis of these two micromodel tests a conclusion has been drawn that models of DA, specular, or mixed types are inappropriate for any other models of condensed systems. This pessimistic point of view closes the way of formulation of model $\left({ }^{2}\right)$ electrodynamics of nonlocal media with arbitrary bulk dielectric functions $[42,45,52]$ and that is the first reason why still some papers based on dielectric approximations appear in the literature. Moreover, it is evident that more complicated systems, such as polar liquids, electrolyte solutions, and other imperfect condensed media cannot be treated on the basis of first-principle micromodels in the broad frequency range : neither the bulk $\varepsilon_{\alpha \beta}(\omega, k)$ response, nor the $\varepsilon_{\alpha \beta}\left(\omega, z, z^{\prime}\right)$ of semi-infinite media can be calculated at present. This make it impossible to probe the form of the constitutive relation (or $\mathrm{ABC}$ ) on the micromodel grounds i. e. to obtain convincing negative results.

From the presented discussion it becomes evident that the only rigorous way of solving the electrodynamical problem in interfacial systems with spatial dispersion is the electromagnetic Green's function formalism, surpassing the problem of definition of dielectric function, i. e. starting from the Dyson equation.

5. Collective excitations and dielectric properties in the bulk of the components of electrochemical systems. - Before discussing the collective phenomena in interfacial electrochemical systems, we have to survey the possible excitations in the bulk of the relevant components.

We recall that the longitudinal collective excitations of polarization fluctuations are described by the dispersion law $[18,31]$

$$
\varepsilon_{||}(\omega, k)=0 \text {. }
$$

The root of this equation provides the dependence of eigenmode $\omega$ on the possible wave-vector $\mathbf{k}$. On the other hand, normal electromagnetic modes in the medium (polaritons) which are the mixture of electromagnetic waves in the medium and transversal excitations of the polarization fluctuations obey the dispersion law

$$
\varepsilon_{\perp}(\omega, k)=k^{2} c^{2} / \omega^{2}
$$

(2) The term model is concerned with the variant of the constitutive relation. 
5.1 METAL ELECTRODES. - In metals, the value of the Fermi-energy is of the order of $10^{4} \mathrm{~K}$, so the electron gas is always degenerate.

Let us consider firstly the excitations possible in a degenerate one-component electron gas disregarding self-frequencies of the background skeleton, periodical structure leading to interband transitions [31] and local field corrections [31] by putting the background dielectric constant equal to $1(5.1)$ with $\varepsilon(\omega, k)$ of degenerate electron plasma [13], for

$$
\hbar k \ll p_{\mathrm{F}}=m V_{\mathrm{F}}
$$

(the Fermi momentum) gives [53].

$k^{2} v_{\mathrm{F}} / 3 \omega_{\mathrm{p}}^{2}=-1+\frac{1}{2} \frac{\omega}{k v_{\mathrm{F}}} \ln \left[\left(\omega+k v_{\mathrm{F}}\right) /\left(\omega-k v_{\mathrm{F}}\right)\right]$.

For $k \ll \omega_{\mathrm{p}} / v_{\mathrm{F}}$, equation (5.3) provides the Vlasov dispersion law [54]

$$
\omega^{2}=\omega_{\mathrm{p}}^{2}+\frac{3}{5} v_{\mathrm{F}}^{2} k^{2}=\omega_{\mathrm{p}}^{2}\left(1+\frac{9}{5} k^{2} / x_{\mathrm{FT}}^{2}\right) .
$$

In the short-wave length limit $h k^{2} / 2 m \ll \omega_{\mathrm{p}} \ll k v_{\mathrm{F}}$, the general equation [13] leads to [55]

$$
\begin{aligned}
\hbar \omega & =\frac{\left(p_{\mathrm{F}}+\frac{\hbar k}{2}\right)^{2}-p_{\mathrm{F}}}{2 m} \\
& \times\left[1+\exp \left(-\frac{2}{3} \frac{v_{\mathrm{F}}^{2} k^{2}}{\omega_{\mathrm{p}}^{2}}\right)\right]^{-2} \approx v_{\mathrm{F}}\left(p-p_{\mathrm{F}}\right)
\end{aligned}
$$

where $p:=\hbar k+p_{\mathrm{F}}$ is the electron momentum in an excited state. Thus (5.4) corresponds to single-particle excitations out of the Fermi-surface of completely degenerate plasma.

The dispersion law (5.5) corresponds to collective excitation of longitudinal polarization waves in degenerate plasma, i. e. plasmons. Thus, dielectric theory

$\begin{array}{lcccc}\text { Material } & \mathrm{Bi} & \mathrm{ZnS} & \mathrm{M}_{0} \mathrm{O}_{3} & \mathrm{SiO}_{2} \\ \hbar \omega_{\mathrm{p}} \mathrm{eV} & \overline{15} & \frac{\overline{17}}{25} & \frac{\overline{25}}{25} & \end{array}$

In slightly doped semiconductors and at room temperatures the plasma is maxwellian. Due to the low concentration of charge carriers, the plasma frequency becomes smaller than any frequency of interband transitions. The solution of equation (5.1) gives now the plasmon dispersion in the range of small $k\left(<\omega / v_{F}\right)$ where damping may be neglected :

$$
\begin{array}{ll}
\omega^{2}=\tilde{\omega}_{\mathrm{p}}^{2}\left(1+k^{2} / \tilde{\varkappa}^{2}\right) & (k \ll \tilde{\varkappa}) \\
\tilde{\omega}_{\mathrm{p}}^{2}=4 \pi N_{\mathrm{c}} e^{2} / \varepsilon m^{*} ; & \tilde{\varkappa}=4 \pi N_{\mathrm{c}} e^{2} / k T \varepsilon .
\end{array}
$$

Plasmons here undergo Landau-damping at wave vectors much smaller than in the case of degenerate plasma, due to the inequality $\tilde{x} \ll x_{\mathrm{TF}}$.

Transversal waves in plasma are possible only for $\omega>\tilde{\omega}_{\mathrm{p}}$. For lower frequencies than $\tilde{\omega}_{\mathrm{p}}$ the semi- with spatial dispersion is capable to cover both the long-range collective motions (plasmons) and the short-range single-particle motions. The region of $k \approx \omega / v_{\mathrm{F}}$ is also incorporated in the general formulas, so that for $k>\omega / v_{\mathrm{F}}, \varepsilon_{\|}(\omega, k)$ has a significant imaginary part (e.g. $\ln \left(\omega-k v_{\mathrm{F}}\right)$ becomes complex) and the waves are heavily damped. This phenomenon is known as Landau-damping in degenerate plasma.

5.2 Semiconductor electrodes, - Firstly, we discuss materials, which can be described in terms of degenerate plasma. These are strongly doped semiconductors and semi-metals. An interesting feature of these compounds and elements (such as InSb, PbTe, and $\mathrm{Bi}$ with the concentration of charge carries not smaller than $10^{18} \mathrm{~cm}^{3}$ ) is that solid state plasma can be considered in the free electron gas model, though the concentration, $\mathrm{Ne}$, is lower than for metals. The Bohr radius should here be replaced by the effective Bohr radius

$$
a_{\mathrm{b}}^{\mathrm{eff}}=\hbar^{2} \varepsilon / m^{*} e^{2}
$$

where $\varepsilon$ is the dielectric constant determined by interband transitions and $m^{*}$ the effective mass. The effective mass in these substances may be smaller than $m$, due to different effects. $\varepsilon$ for the systems with narrow forbidden band may be very large $(\varepsilon \approx 100$ for $B i)$. This is the reason why

$$
a_{\mathrm{b}}^{\text {eff }} \gg a_{\mathrm{b}}
$$

in such kind of materials, which ensures that the condition

$$
N_{\mathrm{e}}^{1 / 3} \gg\left(a_{\mathrm{b}}^{\text {eff }}\right)^{-1}
$$

is satisfied. This situation holds for a set of intrinsic semiconductors. The plasma frequency in some materials, for which the degenrate electron gas model is appropriate, are presented in the table $[30,31]$ :

$$
\begin{array}{lllll}
\mathrm{Al}_{2} \mathrm{O}_{3} & \mathrm{TiO}_{2} & \mathrm{SnO}_{2} & \frac{\mathrm{Si}}{17} & \frac{\mathrm{Ge}}{17}
\end{array}
$$

conductor is nontransparent. Exceptive is the case when modes other than plasmatic are present in the system (see, e.g., combined plasmon-phonon modes $[31,81-81])$.

5.3 Electrolyte solutions. - In the radiowave and microwave frequency ranges, solvent collective motions (Debye fluctuations) are coupled with the fluctuations of the ionic atmosphere (DebyeFalkenhagen effect). At present there are no rigorous theory of these combined collective motions.

At higher frequencies - in the infrared regions, a phonon-like excitation of the solute quasi-lattice are supposed to be found [56] for concentrations of the order of $4 \mathrm{~mol} / \mathrm{dm}^{3}$. The reason for appearance of 
optical phonon-like modes in concentrated electrolyte solution was revealed by neutron diffraction investigations by Enderby and his associates [57]. At the concentrations of $5.5 \mathrm{~mol} . / \mathrm{dm}^{3}$, it was shown that $\mathrm{NiCl}_{2}$ solutions in $\mathrm{D}_{2} \mathrm{O}$ possessed a highly regular arrangement of ions (lattice-like structure). These results were interpreted as a first clear structural evidence in favour of the quasi-lattice picture of concentrated aqueous solutions [58]. Having adopted this concept it was natural to try to find possible excitations in this quasi-lattice. In order to do this, Fontana [56] measured difference Raman spectra (the total solution spectra minus pure solvent spectrum, which was assumed to be unchanged with the variation of concentration of solute).

At a solute concentration of $4 \mathrm{~mol} / \mathrm{dm}^{3}$ the difference spectrum contains a broad peak at $220 \mathrm{~cm}^{-1}$. With decrease of concentration the spectrum broadens (is shifted to lower frequency) and at $1 \mathrm{~mol} . / \mathrm{dm}^{3}$ the difference spectrum disappears. The author associated his result as optical phononlike excitations in a lattice-like structure which is being formed when the concentration value passes from $1 \mathrm{~mol} . / \mathrm{dm}^{3}$. The reliability of the results is questionable in the low frequency wing of the spectrum, since it is difficult to predict the $\mathrm{Ni}^{2+}$ destructuring effect on the hydrogen bonding chains of pure water which increases with the increase of the $\mathrm{Ni}^{2+}$ concentration. Thus, at low frequencies the difference spectrum is not well defined. However, the main problem is whether this spectrum is not associated with the shallow vibration frequencies of ionic pairs coupled by electrostatic forces $\left(\mathrm{Ni}^{2+}+\mathrm{Cl}^{-}\right)$, hindered by the interaction with solvent and the rest of the solute. In this case the number of ionic pairs increases with the increase of concentration, thus, the oscillator strength increases. Following this interpretation of Fontana's experiment, one could assume that at $1 \mathrm{~mol} . / \mathrm{dm}^{3}$ the number of ionic pairs is negligible.

5.4 Molten Salts. - Molten salts based on ionic crystals can behave in two ways in the limits of high and low temperatures. The mean interionic interaction energy $e^{2} N^{1 / 3} / \varepsilon$ should be compared with $k T$. It can be regarded as an ideal plasma (a dense one, with short range repulsion forces) if $e^{2} N^{1 / 3} / \varepsilon_{\infty} k T \ll 1$. In the opposite case, when $e^{2} N^{1 / 3} / \varepsilon_{\infty} k T \gg 1$ one should expect quasicrystalline or liquide-like behaviour of molten salts. Thus, we may evaluate the critical temperature for a fixed volume. Assuming $\varepsilon_{\infty} \simeq 2$, $N^{1 / 3}=10 \AA, \quad T^{*} \approx 7000 \mathrm{~K}$. This temperature is not achieved in electrochemical experiments. Thus, structural and optical properties of molten salts should reveal a certain type of order with corresponding smoothed (but still defined) bands of dipole-active collective excitations. Evidence toward quasi-crystalline order in molten salts has been obtained by different scattering experiments (see e.g. reference [60]).
5.5 Solid eleCtrolytes. - While the structural concepts of solid electrolytes are rather well developed, the nature of their dynamic behaviour is much less clear. We shall confine our discussion by the compounds of $\alpha$-AgI type [61].

The popular model for this system supposes the presence of a rigid sublattice formed by anions (in $\mathrm{AgI}, \mathrm{CuBr}, \mathrm{Cu}_{2} \mathrm{~S}$, etc.) or by anions and immobile cations (in $\mathrm{Ag}_{4} \mathrm{RbI}_{5}, \mathrm{Ag}_{3} \mathrm{SBr}$, etc.). The mobile cations (these are usually, light $\mathrm{Ag}^{+}$or $\mathrm{Cu}^{+}$ions) are assumed to be distributed over the voids in the rigid sublattice. Crystallochemical estimates [61, 62] indicate that the rigid anion arrangement in $\alpha$-AgI provides 21 possible positions for $\mathrm{Ag}^{+}$ion per one $\mathrm{I}^{-}$; and this fact is considered as a reason of strong disordering in $\mathrm{Ag}^{+}$distribution. Due to the collective interaction between mobile cations, these local levels, which are not deep themselves with respect to thermal energy, degenerate into cationic conductivity bands, or at least they become smoothed. These arguments justify the cationic liquid model of solid electrolytes, in which the mobile cations may participate in continuous collective translational motions as well as in shallow oscillations near momentary positions [61].

The infrared spectrum of $\alpha-\mathrm{AgI}$ and $\alpha-\mathrm{Ag}_{4} \mathrm{RbI}_{5}$ was observed in the frequency range of $10^{11}-10^{12} \mathrm{~s}^{-1}$ [63]. It was found that a smoothed resonance is located near the lower frequency side of the usual rather sharp optical phonon resonance. This higher frequency peak is similar to the corresponding peak in the $\beta$-phase (the phase with ordering over both the cations and anions), while the lower frequency absorption band almost disappears in the $\beta$-phase (both peaks almost completely overlap each other). One of the possible explanations of these results is that the higher frequency peak is due to rigid lattice optical vibrations (phonons), while the lower frequency one is caused by the shallow highly anharmonic, but still confined cooperative movements of cations near momentary equilibrium positions. Another possible interpretation of these data starts from the plasmalike behaviour of the cationic subsystem. To describe it, one adopts the jellium model, in which cations are considered to be free and dissolved in a uniform continuum of skeleton dielectric constant $\varepsilon_{\mathrm{s}}(\omega)$. The nonuniformity of the anion skeleton (its difference from the jellium) and cation-cation collisions may be involved by means of effective collision time. $\varepsilon_{\mathrm{s}}(\omega)$ incorporates the phonon-like resonance due to anion rigid sublattice vibrations. The cationic plasma-frequency $\omega_{\mathrm{p}}=4 \pi N_{\mathrm{c}} e^{2} / m_{\mathrm{c}} \approx 10^{11} \mathrm{~s}^{-1}$; thus, cation-plasma oscillations are dressed by vibrations of the skeleton.

6. Surface modes. - At the interfaces of media, possessing bulk collective modes, surface collective modes may arise. Electromagnetic modes, composed of surface oscillations of the medium and electromagnetic waves propagating along the boundary are 
called surface polaritons (SP). The amplitude of the true surface modes decays exponentially along the direction normal to the surface.

Surveys of the SP theory may be found in references $[1,2,79,59,64$ and 80$]$. We shall only summarize some general aspects of SP, which may be useful in the analysis of each mode, which may be excited at electrochemical interfaces.

Surface modes are considered to be well defined, if imaginary part of the wave-vector component along the surface is smaller than inverse distances of penetration of the excitation into the bulk of the contacting media (i. e., the value of the imagining part of the wave-vector component, normal to the surface). On the other hand, if the penetration is still much greater than the characteristic microscopic distances of the interfacial region, the laws of propagation of surface polaritons can be formulated on the basis of phenomenological Maxwell electrodynamics. Since such an approach provides a certain generality to make conclusions on the electromagnetic response of different contact system, we shall again be confined by the framework of the dielectric formalism. (The reviews and articles on the microscopic theory of surface modes, see in references $[1,2,51$, $65-67,79]$ ).

Firstly, we discuss SP, neglecting spatial dispersion. At the plain boundary of two media with $\varepsilon_{\mathrm{a}}(\omega)$ and $\varepsilon_{\mathrm{b}}(\omega)$ surface modes are found as normal solutions of Maxwell equations subject to the boundary conditions. If the media, for simplicity, are regarded as nonmagnetic, isotropic, and with no external charges, these solutions are

$$
\begin{aligned}
& \mathbf{E}_{\mathrm{a}}=\mathbf{E}_{\mathrm{a}}^{0} \mathrm{e}^{i k_{\mathrm{a} \perp z}} \mathrm{e}^{i\left(k_{\|} x-\omega t\right)} y<0 \\
& \mathbf{E}_{\mathrm{b}}=\mathbf{E}_{\mathrm{b}}^{0} \mathrm{e}^{i k_{\mathrm{b}} \perp z} \mathrm{e}^{\left.i k_{\|} x-\omega t\right)} \quad y>0
\end{aligned}
$$

( $z$ is normal to the interface) and they obey the dispersion laws

$$
\begin{aligned}
& k_{\|}^{2}=\frac{\omega^{2}}{c^{2}} \frac{\varepsilon_{\mathrm{a}}(\omega) \varepsilon_{\mathrm{b}}(\omega)}{\varepsilon_{\mathrm{a}}(\omega)+\varepsilon_{\mathrm{b}}(\omega)} \\
& k_{\mathrm{a} \perp}^{2}=k_{\|}^{2} \frac{\varepsilon_{\mathrm{a}}(\omega)}{\varepsilon_{\mathrm{b}}(\omega)} ; \quad k_{\mathrm{b} \perp}^{2}=k_{\|}^{2} \frac{\varepsilon_{\mathrm{b}}(\omega)}{\varepsilon_{\mathrm{a}}(\omega)} .
\end{aligned}
$$

The types of the surface modes can be classified by the analysis of these equations [64].

6.1 NONDISSIPATIVE MEDIA $\left(\operatorname{Im} \varepsilon_{\mathrm{a}}=\operatorname{Im} \varepsilon_{\mathrm{b}}=0\right)$. - 6.1.1 When $\varepsilon_{\mathrm{a}}>0, \varepsilon_{\mathrm{b}}>0, k_{\|}$and $k_{\mathrm{a} \perp}, k_{\mathrm{b} \perp}$ are real, and (6.2), (6.3) correspond to the so-called Brewster solutions. They are not coupled with the surface $\left(k_{\perp}\right.$ is real), but satisfy the condition (eq. (6.1)) that in each medium there is only one wave (incident and transmitted wave with no reflected one); eq. (6.3) defines the values of Brewster angles.

$6.1 .2 \varepsilon_{\mathrm{a}}(\omega)>0, \varepsilon_{\mathrm{b}}(\omega)<0$, but $\varepsilon_{\mathrm{a}}(\omega)+\varepsilon_{\mathrm{b}}(\omega)<0$. Then $k_{\|}$is real, and

$$
k_{\mathrm{a} \perp}=i k_{\|}\left(\left|\frac{\varepsilon_{\mathrm{a}}}{\varepsilon_{\mathrm{b}}}\right|\right)^{1 / 2}
$$

and

$$
k_{\mathrm{b} \perp}=i k_{\|}\left(\left|\frac{\varepsilon_{\mathrm{b}}}{\varepsilon_{\mathrm{a}}}\right|\right)^{1 / 2}
$$

imaginary. These are true surface modes decaying exponentially into the bulk. It is seen from (6.2) that in this case

$$
k_{\|}(\omega)>k_{\text {bulk a }}(\omega) \equiv \frac{\omega^{2}}{c^{2}} \varepsilon_{\mathrm{a}}(\omega)
$$

or

$$
k_{\text {bulk b }}(\omega) \equiv \frac{\omega^{2}}{c^{2}} \varepsilon_{\mathrm{b}}(\omega) .
$$

Thus, surface modes are not mixed with bulk modes, since the former have too large wave-vectors. The medium with $\varepsilon<0$ is, sometimes, called surface active in the sense that, being combined with a medium with $\varepsilon>0$ (in the frequency range under consideration), it produces a system in which the propagation of surface modes is possible.

When the surface inactive substrate is vacuum $\left(\varepsilon_{\mathrm{b}}=1\right)$ the dispersion law is

$$
k_{\|}=\frac{\omega^{2}}{c^{2}} \frac{\varepsilon(\omega)}{\varepsilon(\omega)+1} .
$$

In the limit of large $k_{\|}\left(k_{\|}^{2} c^{2} / \omega^{2} \gg 1\right.$, i. e. in the absence of retardation) the dispersion law is $\varepsilon(\omega) \approx-1$, the solution of which does not depend upon the wavevector, i. e., it corresponds to surface oscillations with group velocity equal to zero. In the opposite, long wave-length limit,

$$
\left(k_{\|}^{2} c^{2} / \omega^{2} \ll 1\right) \quad \varepsilon(\omega) \approx k_{\|}^{2} c^{2} / \omega^{2}
$$

which corresponds to a transversal electromagnetic wave propagating along the $\mathbf{k}$ direction. Thus, SP are combinations of transversal electromagnetic waves and longitudinal surface oscillations.

6.1.3 When both $\varepsilon_{\mathrm{a}}<0$ and $\varepsilon_{\mathrm{b}}<0: k_{\|}, k_{\mathrm{a} \perp}$ and $k_{\mathrm{b} \perp}$ are purely imaginary, and the system is nontransparent for any waves to be excited, neither in the bulk, nor at the surface.

6.2 Dissipative media. - In a system in which one component is dissipative, the solutions of (6.2) and (6.3) for $k_{\perp}$ and $k_{\|}$are complex. Let $\operatorname{Im} \varepsilon_{\mathrm{a}} \neq 0$, $\operatorname{Im} \varepsilon_{\mathrm{b}}=0, \operatorname{Re} \varepsilon_{\mathrm{b}}>0$. If $\varepsilon_{\mathrm{a}}^{\prime}<0$, the case corresponds to dissipative surface modes : the real parts of the $k_{\perp \mathrm{a}}$ and $k_{\perp \mathrm{b}}$ components then have opposite signs. Other possibilities are considered in [64].

SP in the medium with damping are still defined when $\operatorname{Im} k_{\perp} \approx \operatorname{Im} k_{\|} \leqslant \operatorname{Re} k_{\|}$. Let us firstly consider the boundary between the surface active medium and vacuum $\left(\varepsilon_{\mathrm{a}}=\varepsilon ; \varepsilon_{\mathrm{b}}=1\right)$. Then

$$
\begin{aligned}
& \kappa^{2}=k_{\|}^{2} c^{2} / \omega=1-\frac{\varepsilon^{\prime}+1}{\left(\varepsilon^{\prime}+1\right)^{2}+\varepsilon^{\prime 2}} \\
&+i \frac{\varepsilon^{\prime \prime}}{\left(\varepsilon^{\prime}+1\right)^{2}+\varepsilon^{\prime \prime 2}}
\end{aligned}
$$


The imaginary part of $\kappa$ is small compared to the real one, when

$$
\left|\varepsilon^{\prime}\left(\varepsilon^{\prime}+1\right)+\varepsilon^{\prime 2}\right| \gg\left|\varepsilon^{\prime \prime}\right| \text {. }
$$

Another relevant case arises when the surface active medium is nondissipative, but the boundary inactive substrate involves damping.

Then,

$$
\kappa^{2} / \varepsilon=\frac{\left(\varepsilon+\varepsilon^{\prime}\right) \varepsilon^{\prime}+\varepsilon^{\prime \prime 2}}{\left(\varepsilon+\varepsilon^{\prime}\right)^{2}+\varepsilon^{\prime 2}}+i \frac{\varepsilon^{\prime \prime} \varepsilon}{\left(\varepsilon+\varepsilon^{\prime}\right)^{2}+\varepsilon^{\prime \prime 2}} .
$$

Thus, the necessary criterium is

$$
\left|\left(\varepsilon+\varepsilon^{\prime}\right) \varepsilon^{\prime}+\varepsilon^{\prime \prime 2}\right| \gg\left|\varepsilon^{\prime \prime} \varepsilon\right|
$$

6.3 SURFACE POLARITONS WITH SPATIAL DISPERSION. - When the value of $\operatorname{Im} k_{1}$, is comparable to the characteristic wave vector of spatial dispersion of any of the components, the spatial dispersion of its dielectric function cannot be neglected. Sometimes, it leads only to small corrections to the nondispersive values, but usually it gives rise to qualitatively new phenomena, of which we recall the following :

1. Dependence of the surface mode eigenfrequency on the wave vector in the limit of large $k$, when retardation is neglected, and there is a nonzero value of the group velocity $[41,28]$.

2. Breakdown of the frequency gap in the polariton spectrum $[2,19,68]$. Possible coupling between the bulk and surface polaritons.

3. The appearance of surface modes in the systems, where they do not arise, if the spatial dispersion is neglected. These are e. g. surface excitons at the dielectric/metal interface [69]. They cannot be excited at the boundary with an ideal metal, since it has a negative permittivity $\left(\varepsilon \simeq-\frac{\omega_{\mathrm{p}}^{2}}{\omega^{2}}\right)$ in the exciton frequency range. For $\left(\operatorname{Im} k_{\perp}\right)^{-1}$ values comparable with the skinlayer depth, the effect of field penetration into the metal changes the situation. The metal then behaves effectively as a sort of surface inactive substrate, and surface excitons are possible.

The effect of spatial dispersion, which change the screening properties of the system, may be especially important in interfacial systems, since the effects are here defined not by gradual changes in screening properties but by the competition between the response of the components [70].

In sharp surface models, different approaches in the dielectric function $\left(r, r^{\prime}\right)$ representation lead to different SP dispersion laws [71]. A simple dispersion equation was obtained by Fuchs and Kliewer [1]. It was established for a dielectric function $\left(z, z^{\prime}\right)-\left(z-z^{\prime}\right)$ representation, justified for electron gas with specular reflection of electrons from the boundary. This expression leads to many useful qualitative results. The use of its modification to describe metal surface active dielectric contacts or the interaction of adsorbed particles intra-molecular vibrations with the metal- substrate collective modes enabled to study the suspected qualitative effects in analytical form [72].

However, the use of other dispersion laws (the integral relations, obtained on the basis of other $r, r \rightarrow r-r^{\prime}$ hypothesis) usually leads to similar qualitative results [71].

6.4 EFFECTS DUE TO INTERFACIAL, DEFECT LAYERS. If the value of $\left|k_{\perp}\right|^{-1}$ is not much greater than the characteristic width, $d$, of defect region at the interface, the latter can also affect the character of surface modes. When the defect layers are well defined and $d \gg\left|k_{1}\right|^{-1}$ the problem can be roughly reduced to the introduction of the slab geometry, such as in the case of oxide layers. When $d \sim\left|k_{1}\right|^{-1}$, the problem may be regarded in a different manner, either microscopically, or phenomenologially, by the introduction of surface currents.

Sometimes the effects are very significant. This is so, e. g., for the case when the eigen frequency of exitations in the transition layer is located in the heart of the polariton frequency spectrum [73] :

The existence of transition layers separating metal from other components may lead, sometimes, to effects similar to those of spatial dispersion in metal.

7. Collective modes localized at electrochemical interfaces. - Surface collective modes can play a significant role in the momentum and energy transfer processes during radiative or radiationless transitions, taking place at electrochemical interfaces. The possibility of existence of this kind of modes in ES is determined by the arguments discussed above; it should be checked for each combination of components in the given frequency range.

In the ultraviolet range, at the contact of metal electrodes with electrolytes, the occurrence of well defined surface plasmons (modified by electrolyte surface inactive substrates) are possible, if the latter is transparent. If the metal is covered by oxide semiconductor or a dielectric film, surface plasmons are also modified by surface inactive slab substrates. If the electrode is a doped semiconductor surface combined phonon-plasmon modes are possible if only their frequencies correspond to the transparent band in the infrared range of the electrolyte subsystem; in the opposite case these modes are heavily damped. The same is the situation for surface excitons for intrinsic semiconductor electrodes. On the other hand the surface counterparts of the collective oscillations in the bulk of the electrolyte subsystem can be realized only if a nonmetallic electrode is surface inactive in this frequency range, and for metallic electrodes - only due to the effect of spatial dispersion. In both cases they will be damped due to dissipation in the electrolyte subsystem.

One of the most important examples, illustrating the effect of collective excitations in ES, is represented by homogeneous and heterogeneous charge transfer 
reactions. This fact was realized by Libby [74], Frank and Platzman [75], etc. The authors have shown that fluctating solvent polarization plays the dynamic role in elementary acts of chemical reactions, providing the overcoming of the Frank-Condon barrier. Recently, it has also been shown [76], that another collective mode of polarization plays an important role, namely the fluctuations in the solute ionic atmosphere. In the modern theory of the elementary act of charge transfer processes [37] it has been shown that the collective effects can be quantitatively described in terms of the corresponding Green's functions (of the operators of the solvent polarization, or that of the ionic atmosphere). Within the framework of this theory, the following different effects were considered : light absorption in solution [77], and vibrational relaxation of impurity molecules, and highly exothermic processes [78]. The first two will be briefly discussed below. For exothermic reactions the theory also provided a law of distribution of the total heat of reaction over different elementary excitations in the system.

As noted in section 2, for the analysis of different linear optical phenomena it is sufficient to find the electromagnetic Green-function $D^{\mathrm{R}}$. In the long-wave length limit (as well as in the cases indicated in section 2) the polarization operator (and, thereby $D^{\mathrm{R}}$ ) can be found from the equation

$$
\pi_{\alpha \beta}\left(\mathbf{r}, \mathbf{r}^{\prime}, \omega_{n}\right)=\left\langle T_{\tau} \dot{P}_{\alpha}(\mathbf{r}, \tau) \dot{P}_{\beta}\left(\mathbf{r}^{\prime}, 0\right)>_{\omega_{n}}\right.
$$

$\dot{\mathbf{P}}(\tau)$ is the operator of current density in the temperature Heisenberg representation [7], $T_{\tau}$ is the temperature chronological operator [7]. $\dot{P}$ includes all the currents in the system : electron current in electrode material and in the molecules of the solvent and impurities (solute) ; intra-molecular vibration current, and the current due to rotational and translational diffusion. All these modes contribute to $\pi$ in different frequency ranges. We shall be concerned below only with infra-red and visible regions, and thus dipole rotations and ionic translations will be neglected.
Using the Born-Oppenheimer approximation, the electronic movements can be separated from the nuclear coordinates (intra-molecular vibrations, infrared oscillation of solvent polarization, phonons in electrode), which can be described in the harmonic approximation (see section 3 ). The main difficulties arise in the theory in connection with an adequate description of the electron subsystems. The most simple case is that of electrons in solvent molecules.

For solvent electron polarization modes the approximation of effective oscillators (quasi-particles) can be used. The use of the random phase approximation (RPA) for electrons in metals and semiconductors leads to analogous representations of the electron polarization in electrode materials (bulk and surface plasmons or excitons). For the localized electrons of the impurities, the problem can be solved in two approximations the first of which is the case when the energy level of the localized electron is situated in the transparent range in the spectrum of the collective excitations of the rest of the system; here the adiabatic approximation should be employed. The second possibility is realized in large impurity molecules with quasi-continuous energy spectra, where RPA is appropriate for the description of electrons delocalized within the impurity.

What was said above is valid for both impurities in the bulk and adsorbed at the interface. Thus, the situation when the photo-transition is associated with an electron providing a chemical bonding of an adatom with the surface $\left(^{3}\right)$ lies beyond of our consideration.

It will be seen from the following that the problem is solved accurately when all the electrons can be described within RPA. Now we discuss the more realistic second possibility. The electrons with energies much greater than that of the level localized at the impurities are only dress for the localized electrons' movements. Let us then consider different contributions to $\pi$. The impurity current is composed of a component due to electronic transitions between different electronic states $m$ and $n$, i. e.

$$
\begin{aligned}
\pi_{\alpha \beta}^{(\mathrm{e})}\left(\mathbf{r}, \mathbf{r}^{\prime}, \tau-\tau^{\prime}\right)=\sum_{n, m} \exp \left(\frac{F-F_{n}}{k T}\right)\left(j_{\alpha}^{(\mathrm{e})}(\mathbf{r})\right)_{n, m}\left(j_{\beta}^{(\mathrm{e})}\left(\mathbf{r}^{\prime}\right)\right)_{m, n} \times \\
\\
\quad \times \operatorname{Sp} \exp \left(\frac{F_{n}}{k T}\right) \exp \left\{-\frac{\mathfrak{J}_{n}}{k T}+\left(\tau-\tau^{\prime}\right) \mathscr{H}_{n}\right\} \exp -\left(\tau-\tau^{\prime}\right) \mathfrak{H}_{m} .
\end{aligned}
$$

Here, $\mathcal{H}^{n}$ is the adiabatic vibrational Hamiltonian describing the state of the medium and the impurity for the given electronic state $n: \exp \left\{\left(F-F_{n}\right) / k T\right\}$ defines the probability of the system to occupy the electronic term $n ;\left(j^{(\mathrm{e})}\right)_{n m}$ is the matrix element for a dipole transition of the impurity. The expression under the Sp sign determines the transition probability between the electronic terms $n$ and $m$; its calculation is described in reference $[37,77,78]$. In these papers only the equilibrium nuclear coordinate displacement was taken into account and frequency shifts in the course of the transition disregarded; this is a common assumption in the theory of radiationless transitions. The latter effect is significant in the theory of vibrational transition.

(3) Such problems have been considered, using RPA, in a paper by Brodsky and Urbakh [11]. 
The vibrational contribution to the polarization operator is

$$
\begin{aligned}
\pi_{\alpha \beta}^{(\mathbf{v})}\left(\mathbf{r}, \mathbf{r}^{\prime}, \tau-\tau^{\prime}\right)=\sum_{n} \exp \left(\frac{F-F_{n}}{k T}\right)\{< & T_{\tau} \dot{P}_{\alpha}^{n}(\mathbf{r}, \tau) \dot{P}_{\beta}^{n}\left(\mathbf{r}^{\prime}, \tau^{\prime}\right)>+ \\
& +<T_{\tau} \dot{P}_{\alpha}^{n}(\mathbf{r}, \tau) \dot{Q}_{j}^{n}\left(\tau^{\prime}\right)>V_{\beta j}^{n}\left(\mathbf{r}^{\prime}\right)+V_{\alpha j}^{n}(\mathbf{r})<T_{\tau} \dot{Q}_{j}^{n}(\tau) \dot{P}_{\beta}^{n}\left(\mathbf{r}^{\prime}, \tau^{\prime}\right)> \\
& \left.+V_{\alpha j}^{n}(\mathbf{r})<T_{\tau} \dot{Q}_{j}^{n}(\tau) \dot{Q}_{j^{\prime}}^{n}\left(\tau^{\prime}\right)>V_{\beta j^{\prime}}^{n}\left(\mathbf{r}^{\prime}\right)\right\} .
\end{aligned}
$$

Here $Q_{j}^{n}$, is the $j$-mode normal coordinate and $\dot{\mathbf{P}}^{n}$ the polarization current vector provided that the impurity particle occupies the $n$-th electronic term;

$$
V_{\alpha j}^{n}(\mathbf{r})=\left(\partial j_{\alpha}^{(n)}(\mathbf{r}, Q)\right)_{n m} /\left.\partial \dot{Q}_{j}^{n}\right|_{Q_{j}^{n}=0}
$$

where $j^{(n)}$ is the impurity nuclear component of the current density.

The first term in equation (7.3) describes correlation of polarization fluctuation in space and time corresponding to the $n^{\prime}$ th electronic state of the impurity. The last term describes the vibrational relaxation of impurity particles. The second and the third terms determine cross-effects of excitation of impurity oscillation by the fluctuations of the polarization of the medium and vice versa.

The temperature Green-functions

$$
\begin{aligned}
G_{\alpha \beta}^{n}\left(\mathbf{r}, \mathbf{r}^{\prime}, \tau-\tau^{\prime}\right) & =<T_{\tau} P_{\alpha}^{n}(\mathbf{r}, \tau) P_{\beta}^{n}\left(\mathbf{r}^{\prime}, \tau^{\prime}\right)>; \\
\mathcal{G}_{\alpha j}^{n}\left(\mathbf{r}, \tau-\tau^{\prime}\right) & =<T_{\tau} P_{\alpha}^{n}(\mathbf{r}, \tau) Q_{j}^{n}\left(\tau^{\prime}\right)> \\
g_{j j^{\prime}}^{n}\left(\tau-\tau^{\prime}\right) & =<T_{\tau} Q_{j}^{n}(\tau) Q_{j^{\prime}}^{n}\left(\tau^{\prime}\right)>
\end{aligned}
$$

which are immediately connected with Green-functions, involved in equations (7.3), can be found from the Dyson equation. In tensor form,

$$
\begin{gathered}
G^{n}\left(\omega_{k}\right)=G^{0}\left(\omega_{k}\right)+G^{0}\left(\omega_{k}\right) V^{n} g^{n}\left(\omega_{k}\right) V^{n} G^{0}\left(\omega_{k}\right) \\
G^{n}\left(\omega_{k}\right)=G^{0}\left(\omega_{k}\right) V^{n} g^{n}\left(\omega_{k}\right) \\
g^{n}\left(\omega_{k}\right)=\left[G^{0-1}\left(\omega_{k}\right)-U\left(\omega_{k}\right)\right]^{-1}= \\
=G^{0}\left(\omega_{k}\right)\left[1-G^{0}\left(\omega_{k}\right) U\left(\omega_{k}\right)\right]^{-1}
\end{gathered}
$$

where

$$
\mathbf{v}_{j}^{n}(\mathbf{r})=\partial \mathbf{E}^{n}(\mathbf{r}, Q) /\left.\partial Q_{j}^{n}\right|_{Q_{j}^{n}=0} .
$$

Here, $\mathbf{E}^{(n)}(\mathbf{r}, Q)$ is the electric field vector in the point $\mathbf{r}$ created by an impurity with electronic state $n$.
$U$ is the renormalized interaction between the different vibrational intra-molecular (impurity) modes via the fluctuation of polarization of the medium :

$U_{j j^{\prime}}^{n}\left(\omega_{k}\right)=\sum_{\alpha, \beta} \int \mathrm{d} \mathbf{r} \mathrm{d} \mathbf{r}^{\prime} V_{\alpha j}^{n}(\mathbf{r}) G_{\alpha \beta}^{0}\left(\mathbf{r}, \mathbf{r}^{\prime} ; \omega_{k}\right) V_{\beta j^{\prime}}^{n}\left(\mathbf{r}^{\prime}\right)$

$\pi$ presented above correspond to the case when there is only one impurity particle in the system. In the limit of high dilution the result is multiplied by its concentration (in the case of impurities adsorbed at the interface the multiplier is the surface concentration). Moreover, $\pi$ should be averaged over possible orientations of the impurity molecules. In the bulk of the solution this averaging is trivial, since $\pi$ does not depend on the orientation in isotropic systems. Much more complicated is the situation for impurity molecules near interfaces. Here, the cases of physical adsorption and chemisorption should be considered separately. In the case of chemisorption one should distinguish the situations when the molecule is chemically bound to the electrode surface by one atom, two atoms, or three atoms (not located on one line). In each of these cases the impurity possesses a different number of orientational degrees of freedom $(1,2$ or 3$)$.

In order to perform the orientational averaging, one has to calculate correlators of the type $\langle v v\rangle$, $\langle v V\rangle,\langle V V\rangle$. These correlators can be found, if the multipole structure of the molecule is known. It should be noted that some modes of orientational movements are not free, but depend on the electrode potential. Therefore, the optical signals contain the information on the applied potential (e. g., via the dependence of $\pi$ upon the orientation angle, which itself depends on the voltage).

\section{References}

[1] KLIEver, K. L. and Fuchs, R., Adv. Chem. Phys. 27 (1974).

[2] Agranovich, V. M., Usp. Fiz. Nauk 115 (1975) 199.

[3] Burstein, E. and De Martini, F., eds., Polariton, Proc. Taormina Res. Conf. on the Structure of Matter (Pergamon, New York) 1974.

[4] Bendow, B., Birman, J. A. and Agranovich, V. M., eds., Theory of Light Scattering in Condensed Matter, Proc. I U.S. A.-U. S. S. R. Symp. (Plenum) 1977.

[5] Delahay, P. and Tobias, C., eds., Adv. in Electrochem. Eng. 9 (1973).

[6] Berne, B. J. and Pecora, R., Dynamic Light Scattering (Wiley-Interscience, New York) 1976.
[7] Abricosov, A. A., Gorkov, L. P. and Dzyaloshinskit, I. E., Methods of Quantum Field Theory in Statistical Physics (GIFML, Moscow) 1962.

[8] Feynman, R. P. and Hibbs, A. R., Quantum Mechanics and Path Integrals (McGraw-Hill, New York) 1965.

[9] Mills, D. L. and Maradudin, A. A., Phys. Rev. B 12 (1975) 2943.

[10] Mills, D. L. and Maradudin, A. A., Phys. Rev. B in press.

[11] Brodsky, A. M. and UrbakH, M. I., Phys. Status Solidi $(b)$, 83 (1977) in press.

[12] Born, M. and Wolf, E., Principles of Optics (Pergamon, Oxford) 1964. 
[13] Silin, V. P. and Rukhadze, A. A., Electromagnetic Properties of Plasma, Atomisdat (Moscow) 1961.

[14] Zubarev, D. N., Nonequilibrium Statistical Thermodynamics (Nauk, Moscow) 1971.

[15] March, N. H., Young, W. H. and Sampanthar, S., The Many Body Problems in Quantum Mechanics (Cambridge, University Press) 1967.

[16] Thouless, D. J., The quantum mechanics of many-body systems (New York, Academic Press) 1961.

[17] Bonch-Bruevich, V. L. and Tyablikov, S. V., Methods of Green's Functions in Statistical Mechanics (GIFML, Moscow) 1961.

[18] Agranovich, V. M. and Ginzburg, V. L., Spatial Dispersion in Crystal Optics and the Theory of Excitons (Interscience, New York) 1966.

[19] Maradudin, A. A. and Mills, D. L., Phys. Rev. B 7 (1973) 2787.

[20] Reuter, G. E. M. and Sondheimer, E. H., Proc. Roy. Soc. A 135 (1948) 336.

[21] Dogonadze, R. R., Kornyshev, A. A. and Kuznetsov, A. M., Teor. Mat. Fiz. 15 (1973) 127 (Eng. trans. Theor. Math. Phys. (USSR) 15 No. 1 (1973)).

[22] Takeno, S. and Goda, M., Progr. Theor. Phys. 47 (1972) 730.

[23] Kim, K. and Nelkin, M., Phys. Rev. B 7 (1973) 2762.

[24] LuCAS, A. A. and ŠunJIČ, M., Progr. Surf. Sci. 2 (1972) 75.

[25] Devreese, J., Evrard, R. and Kartheuser, E., in Elementary Excitations in Solids, Molecules and Atoms, Part B, Devreese, J. T. et al., eds. (Plenum Press, London) 1974.

[26] LuCAs, A. A., ibid. (Part A).

[27] Kharkats, Yu. I., Kornyshev, A. A. and VorotyntsEv, M. A., J. C. S. Faraday Trans. II 72 (1975) 361.

[28] Davydov, A. S., Solid State Theory (Nauka, Moscow) 1976.

[29] For a review, see e. g. $\$ 7$ of ref. [13].

[30] Pines, D. and Noziżres, P., The Theory of Quantum Liquids (Benjamin, New York) 1966.

[31] Platzman, P. M. and Wolff, P. A., Solid State Physics, Suppl. 13 (Academic Press, New York) 1973.

[32] Lundquist, B. I., Phys. Kondens. Mater. 6 (1967) 193 ; 7 (1968) 117.

[33] Inkson, J. C., J. Phys. C: Solid State Phys. 5 (1972) 2599.

[34] Lipari, N. O., Phys. Status Solidi (b) 45 (1971) 117.

[35] Shultze, K. R. and Unger, K., Phys. Status Solidi (b) 66 (1974) 491.

[36] Dogonadze, R. R. and Kornyshev, A. A., Phys. Status Solidi (b) 53 (1972) 439.

[37] Dogonadze, R. R. and Kuznetsov, A. M., Prog. Surf. Sci. 6 (1975) 1.

[38] Active Solid State Surfaces Distler G. I. and Butyagin P. Yu., eds. (Nauka, Moscow) 1976 (in Russian).

[39] Heinrichs, J., Phys. Rev. B 8 (1973) 1346.

[40] Pekar, S. I., Zh. Eksp. Teor. Fiz. 33 (1957) 1022.

[41] Hopfield, J. J. and Thomas, D. G., Phys. Rev. 132 (1963) 563.

[42] See $\$ 17$ of ref. [13].

[43] Dingle, R. B., Physica 19 (1953) 311.

[44] Rezvov, A. V., Zh. Tekh. Fiz. 46 (1976) 2479 ; 45 (1975) 2322.

[45] Fuchs, R. and KLIEWER, K. L., Phys. Rev. 172 (1968) 607.

[46] Jones, W. E., KlieWer, K. L. and Fuchs, R., Phys. Rev. 178 (1969) 1201 .

Fuchs, R. and KlIewer, K. L., Phys. Rev. 185 (1969) 905.

[47] Fischer, B, and QueIsSER, H. J., Solid State Commun. 16 (1975) 1125 ; Crit. Rev. Solid State Sci. 5 (1975) 281.

[48] Heinrichs, J., Phys. Rev, B 7 (1973) 3487.

Agarwal, G., Pattanyak, D. M. and Wolff, E., Opt. Commun. 4 (1971) 225 ; 4 (1971) 260 ; Phys. Rev. Lett. 27 (1971) $1022 ; 40 \mathrm{~A}(1972) 279$.

[49] Johnson, D. L. and Rimbey, P. R., Phys. Rev. B 14 (1976) 2398.

[50] Agranovich, V. M., Yudson, V. I., Opt. Commun. 7 (1973) 121.
[51] Sipe, J. E. and van Kranendonk, J., Can. J. Phys. 53 (1975) 2095 ;

Mead, C. A., Phys. Rev. B 15 (1977) 519.

[52] Foley, J. T. and Devaney, A. J., Phys. Rev. B 12 (1975) 3104 ;

Ritchie, R. H. and MARusak, A. L., Surf. Sci. 4 (1966) 234.

[53] Goldman, I. I., Zh. Eksp. Teor. Fiz. 17 (1947) 681.

[54] Vlasov, A. A., Zh. Eksp. Teor. Fiz. 8 (1938) 291.

[55] Kimontovich, Yu. L. and Silin, V. P., Zh. Eksp. Teor. Fiz. 23 (1952) 151.

[56] Fontana, M. P., Solid State Commun. 18 (1976) 765.

[57] Enderby, J. E., Howells, W. S. and Howe, R. A., Chem. Phys. Lett. 21 (1973) 109.

[58] Howe, R. A., Howells, W. S. and Enderby, J. E., J. Phys. $C$ : Solid State Phys. 7 (1974) L111.

[59] Ritchie, R. H., Surf. Sci. 34 (1973) 1.

[60] Edwards, F. G., Enderby, J. E., HowE, R. A. and PAGe, D. I., J. Phys. C : Solid State Phys. 8 (1975) 3483.

[61] Funke, K., Prog. Solid State Chem. II, N 4 (1976) 345.

[62] UKshe, E. A. and BuKUn, N. G., Electrokhimiya 8 (1972) 163.

[63] Eckold, G. and Fumke, K., Z. Naturforsch. 28a (1973) 1042 ;

Gallagher, D. and Klein, N. V., J. Phys. C : Solid State Phys. 9 (1976) L 687 ;

Funke, K. and Jost, A., Ber. Bunsenges. Physik. Chem. 75 (1971) 436.

[64] Burstein, E., Harstein, A., Schoenwald, J., MaraDudin, A. A. and Mills, D. L., see ref. [3], p. 89.

[65] Agranovich, V. M., Mal'shukov, A. G. and MekHTIEv, M. A., Fiz. Tverd. Tela 14 (1972) 849 ; Zh. Eksp. Teor. Fiz. 63 (1972) 2274.

[66] Philpott, M. R., Phys. Rev. B 14 (1976) 3471 ; Mahan, G. D., Obermater, R. G., Phys. Rev. $183(1969) 834$.

[67] Elson, J. M. and RITCHIE, R. H., Phys. Rev. B 4 (1971) 4129.

[68] Bryksin, V. V., Firsov, Yu. A., Fiz. Tverd. Tela 11 (1969) $2167 ; 14$ (1972) 1148.

[69] HeinRIChs, J., Phys. Rev. B 8 (1973) 1708 ;

MaL'shukov, A. G., Dissertation, Institute of Spectroscopy (Acad. Sci. USSR), Moscow, 1974.

[70] Kornyshev, A. A., Rubinshtein, A. I. and VoroTYNTSEv, M. A., Phys. Status Solidi (b) (1977) to be published.

[71] Rimbey, R., Phys. Rev. B 15 (1977) 1215.

[72] MaL'shukov, A. G., private communication.

[73] Agranovich, V. M. and Mal'shukov, A. G., Opt. Commun. 11 (1974) 169.

[74] Libis, W. F., J. Chem. Phys. 56 (1952) 863.

[75] Platzmann, R. and Frank, J., Z. Phys. 138 (1954) 411

[76] Dogonadze, R. R. and Kuznetsov, A. M., J. Electroanal. Chem. 65 (1975) 545.

[77] Dogonadze, R. R., Itskovich, E. M., Kuznetsov, A. M. and Vorotyntsev, M. A., J. Phys. Chem. 79 (1975) 2827.

[78] Dogonadze, R. R., Kuznetsov, A. M. and VorotyntSEv, M. A., Z. Phys. Chem. (N. F.) 100 (1976) 1.

[79] Bryksin, V. V., Mirlind, D. N. and Firsov, Yu. A., Usp. Fiz. Nauk 113 (1974) 29.

[80] Mahan, G. D., in Elementary Excitation in Solids, Molecules and Atoms, part B, p. 93 (Plenum Press, London) 1974.

[81] Mooradian, A. and Wright, G. B., Phys. Rev. Lett. 16 (1966) 999 ;

Mooradian, A. and McWhorter, A. L., Proc. Int. Conf. Light Scattering Spectra in Solids, p. 297 (Springer, New York) 1969.

[82] Buchner, S. and Burstein, E., Phys. Rev. Lett. 33 (1974) 908.

[83] Zemski, V. I., Ivchenko, E. L., Mirdin, D. N. and Reshina, I. $Y$., in ref. [4].

[84] Baumard, J. F. and Gervais, F., Phys. Rev. B 15 (1977) 2316 ; Solid State Commun. 21 (1977) 861.

[85] Singha, S. K., Gupta, R. P. and Price, D. L., Phys. Rev. B 9 (1974) 2564. 


\section{DISCUSSION}

Epelboin. - Au début de votre exposé vous avez évoqué les travaux du Pr. Damaskin de Moscou sur la structure de la double couche électrochimique qui suppose l'existence d'une monocouche d'eau. Comment expliquez-vous que les mesures de la capacité de cette double couche effectuées tant sur une électrode à goutte de mercure de type Heyrovski que sur des électrodes métalliques solides aboutissent à la même structure de la double couche, malgré des différences fondamentales dans l'état de surface de ces deux types d'électrodes?

DogonADZE. - The double layer structure depends primarily on the interaction forces between the metal and the water molecules. If these forces are of the same nature and magnitude one might well expect the same double layer structure for both liquid and solid state electrodes.

EPELboin. - L'analogie entre ces deux types d'électrode est un peu formelle car elle provient à mon avis du fait que les mesures d'impédances électrochimiques sont limitées aux fréquences supérieures à plusieurs Hertz. Avec les électrodes à goutte de mercure du type Heyrovski cette limitation est due au renouvellement constant de la surface métallique et avec les électrodes solides au caractère inductif que présente aux fréquences plus basses l'impédance électrochimique des électrodes métalliques. Ce caractère inductif s'explique par des processus de relaxation des intermédiaires adsorbés sur la surface du métal et peut être étudié également à l'aide des impédances électro-optiques. Avez-vous des données théoriques sur ces processus de relaxation très basse fréquence?
DogonAdze. - Relaxation times of this low order of magnitude may be associated with several kinds of processes generally relating to chemical conversions such as kinetically controlled adsorption. This might be incorporated in a theoretical framework, but the properties of the system must be specified further before you can ascribe these relaxation times to specific phenomena.

YEAGER. - I question the validity of evaluating the optical dielectric constants and thickness of mono and submonolayers of an adsorbed species on a metal surface using ellipsometry and reflectance techniques. The optical properties of the interface are collective properties and not just those of the monolayer. The formation of the monolayer perturbs the optical properties of the surface of the substrate and ambient phase (electrolyte) and these changes must also be considered. Theoretical treatments are needed that treat the optical dielectric properties of the interfaces (with and without various layers) as a whole.

DOGONADZE. - The dielectric permittivity is generally a measure of the response of a given system to an external electric field. As such a dielectric permittivity may be associated with any system, microscopic or macroscopic, and optical measurements may provide information about this function. However, when theoretically relating the dielectric permittivity to microscopic properties of the interphase it is of course necessary to account for all the effects contribution to the interfacial response. 